\title{
Reaction time to the start and end of noise as a function of rise-decay time
}

\author{
SIMON KEMP \\ University of Canterbury, Christchurch, New Zealand
}

\begin{abstract}
Reaction time to the start and end of white noise was measured as a function of rise-decay time. Six values of rise-decay time $(1,5,25,50,100$, and $500 \mathrm{msec}$ ) and two noise levels (40 and $60 \mathrm{~dB}$ SPL) were employed. The results showed reaction time generally to increase with risedecay time and decrease with level. For the shorter rise-decay times, reaction time was faster to the start than to the end of the noise but, for longer rise-decay times, the reverse was true. It is suggested that, when long rise-decay times are used in reaction time experiments, consideration be given to the definition of signal onset.
\end{abstract}

Experiments that compare reaction times to different auditory stimuli have yielded important information on the nature of the auditory process. One such experiment compares reaction time to the start of a sound with that to its end. Generally, the result has been a faster reaction time to the start of sound than to its end (Emmerich, Pitchford, \& Becker, 1976; Goldstone, 1968; Kemp \& Irwin, 1979), a result interpreted by Kemp and Irwin as supporting the notion that the growth of auditory sensation is faster than its decay.

One experiment, however, showed a faster reaction time to the end of a sound than to its start (Grier, 1966a). Generally, the three studies showing a faster reaction time to the start differed in procedure and stimuli among themselves as much as they did from Grier's experiment. However, while the experimental parameters differed widely in these studies, there was one consistent difference between Grier's study and the other experiments: Grier used stimuli with relatively long (100-msec) rise-decay times, whereas the other experiments employed relatively short ones, ranging from 5 microsec to $3 \mathrm{msec}$.

The results of these experiments, then, suggest the possibility of an interaction between rise-decay time and the start or end of a sound with respect to reaction time. Sticht and Foulke (1966) found just such a result from electrocutaneous stimuli. Warm and Foulke (1970), on the other hand, found the opposite result when they considered the effects of rise-decay time on reaction time to the start and end of a $30-\mathrm{dB}$ SL, $1000-\mathrm{Hz}$ tone: that is, with a long rise-decay time $(250 \mathrm{msec})$, the start was responded to more quickly, whereas at short risedecay times ( $\leqslant 5 \mathrm{msec}$ ), there was no difference in reaction time. Two features of Warm and Foulke's experi-

I am grateful to M. B. Simmonds for his useful suggestions on an earlier draft of this paper. The research was partially supported by the National Acoustics Centre of New Zealand. My mailing address is: Department of Psychology, University of Canterbury, Christchurch 1, New Zealand. ment, however, suggest that their result may not be readily generalizable. First, sudden switching (whether on or off) of a pure tone produces audible transients. Warm and Foulke's finding of no difference in reaction time for short rise-decay times may have been caused by subjects' responding to the transients rather than to the start or end of the tone. Experimentally, this problem can be avoided either by using white noise as the stimulus or by masking the transients with white noise. Second, reaction time to the start of the tone increased very little with increasing rise time $-20 \mathrm{msec}$ as the rise time was increased from 25 to $250 \mathrm{msec}$. This result is contrary to the results of other experiments: With increasing rise time, Grier (1966b) and Santee and Kohfeld (1977) obtained a much faster increase in reaction time to the onset of sound.

\section{METHOD}

\section{Subjects}

Five male and five female volunteers, all with normal hearing, served as subjects throughout the experiment. The subjects were paid for participating; none had previously taken part in an auditory reaction time experiment.

\section{Apparatus}

White noise was generated and fed into a Grason-Stadler $829 \mathrm{E}$ electronic switch. The noise was attenuated to the desired level and presented to the subject monaurally through a TDH-49 earphone. The subjects were seated in a sound-attenuating chamber, where they responded by means of a telegraph key on a low table to the right (left for left-handers). The time taken to react to the start or end of the noise was recorded on an electronic counter connected to the electronic switch and telegraph key.

\section{Procedure}

The subjects listened to the sound with the preferred ear. They were requested to respond as quickly as possible by depressing the telegraph key to the occurrence of an auditory event. Three stimulus parameters were varied in the experiment. (1) Type of event: The subjects listened for either the onset of the white noise (in quiet) or the cessation of the white noise. In both cases, the subjects were asked to respond as soon as they perceived a change and not to wait until the noise had reached 
its apparent maximum level or, conversely, until the noise had completely vanished. (2) Sound pressure level of the noise: Two levels, 40 and $60 \mathrm{~dB}$ SPL, were employed. (3) Rise-decay time of the noise: The times used were $1,5,25,50,100$, and $500 \mathrm{msec}$, measured oscilloscopically as the time between the points of $10 \%$ and $90 \%$ of maximum voltage. (The actual calibration was carried out using a deterministic signal rather than the white noise.)

Each trial consisted of the subject's responding to the occurrence of an event. The subject's response terminated the event in the start (end) condition, the noise was turned off (on) by the subject's response.

It is necessary to define the start and end of the signal carefully. The envelope voltage produced by the switch is approximately linear with time between the points at which $10 \%$ and $90 \%$ of maximum envelope voltage is reached. Outside this range, however, the function is flattened: envelope voltage changes more slowly with time. Thus, particularly for long rise-decay times, there is a gap between the triggering of the electronic switch and the commencement of the start or end of the noise signal. For the reaction times reported here, the start of the noise is defined from the point at which the envelope voltage reached $10 \%$ of its maximum level, and the end of the noise, from the $90 \%$ point. The switch reduced voltage in its decay mode at the same rate at which it increased voltage in the rise mode.

The combination of stimulus parameters (event type, level, and rise-decay time) was held constant during blocks of 17 trials. The subjects were warned beforehand of the types of stimuli to which they would be responding. The first two trials of each block were used as practice, and their reaction times were discarded. The subjects were allowed a short pause of about 20 sec between each block; the duration of the pause was signaled by a rest light. There were longer pauses between each group of three blocks. Twelve blocks were run in a session, which lasted about $30 \mathrm{~min}$.

Each of the subjects participated in one practice and four experimental sessions. During each experimental session, the level of the noise was held constant; half the subjects began with the 40-dB SPL noise, and half, with the 60-dB SPL noise. An ABBA design determined the noise levels for the remaining sessions. In the first " $A$ " (or "B") session, the order of presentation of the experimental combinations of event type and risedecay time was chosen randomly and then reversed in the second "A" (or "B") session. Each combination of the three experimental parameters was thus presented twice to each subject.

\section{RESULTS AND DISCUSSION}

The median, rather than the mean, reaction time for each block was extracted in order to reduce the effect of possible contamination from very short (indicative of anticipation) and very long (indicative of lapses of concentration) reaction times. Trials resulting in very long reaction times $(>1 \mathrm{sec})$ were repeated. This occurred rarely. Trials resulting in very short reaction times $(<150 \mathrm{msec})$ were not repeated; only eight such trials occurred throughout the experiment.

Analysis of variance showed that reaction times did not vary significantly between the first and second presentations of a set of stimulus conditions $[F(1,9)=3.69$, $n . s$.$] . There were also no significant (p<.01)$ interactions between presentation order and any of the stimulus parameters or combinations of them. Thus, there appears to be no significant contamination of the data by order effects between sessions. Data from the two sessions were averaged for the results presented below.
There was considerable between-subject variability: median reaction times, averaged over all conditions and sessions, ranged from 230 to $334 \mathrm{msec}$.

Figure 1 shows the median reaction time for each stimulus condition, averaged over the 10 subjects and two blocks as a function of rise-decay time. Reaction times for different events and noise levels are shown separately. Figure 1 summarizes the results, whose significance is confirmed by analysis of variance. There were significant main effects of level $[F(1,9)=289.25$, $\mathrm{p}<.01]$ and rise-decay time $[\mathrm{F}(5,45)=109.58, \mathrm{p}<.01]$; reaction times decreased with increasing noise level and increased with increasing rise-decay time. Both of these results confirm the results of earlier experiments (Grier, 1966a; Murray, 1970; Santee \& Kohfeld, 1977; Warm \& Foulke, 1970) and extend the generality of the results to cover noise as well as tonal stimuli. Overall, there was no significant effect of event type $[F(1,9)=5.64$, n.s. $]$.

Analysis of variance revealed that three of the four possible interaction effects were significant: level $\mathrm{x}$ event type $[F(1,9)=245.29, p<.01]$, level $x$ rise-decay time $[F(5,45)=20.20, p<.01]$, and level $x$ event type $x$ risedecay time $[F(5,45)=44.45, p<.01]$. The interaction of event type $\mathrm{x}$ rise-decay time was not significant $[F(5,45)=1.21$, n.s. $]$. Probably the easiest, and most theoretically relevant, way to interpret this complex tangle of interactions is to consider the results obtained with the two noise levels separately. With the lower noise level (40 dB SPL), the results show, first, that the reaction time to the start of the noise increased rapidly with rise-decay time, essentially replicating the results of Grier (1966b) and Santee and Kohfeld (1977). Second, at short rise-decay times ( 1 and $5 \mathrm{msec}$ ), the start of the noise was responded to faster than its end, while at long rise-decay times (100 and $500 \mathrm{msec})$, the end of the noise was responded to faster. That is, the result discussed in the introduction was obtained. On the other hand, with the higher noise level ( $60 \mathrm{db}$ SPL), (1) the reaction time to the start of the noise increased slowly, if at all, with rise-decay time, and (2) with increasing rise-decay time, the start of the noise was responded to relatively faster than the end. That is, with the exception of the fact that at short rise-decay times

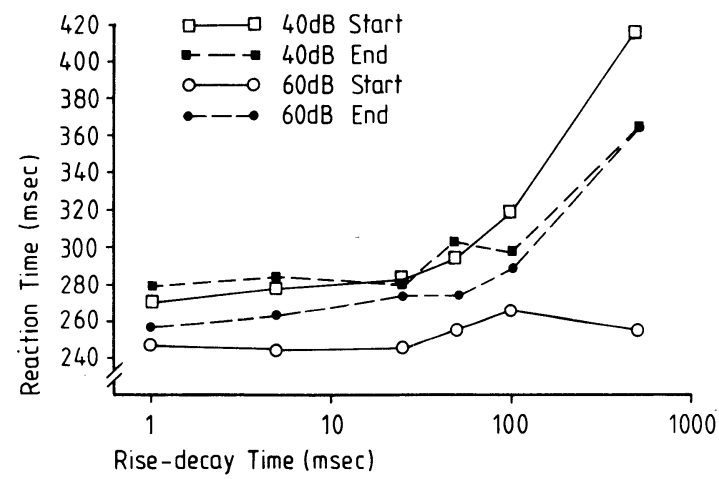

Figure 1. 
the present data showed faster responses to the start of the sound, the results at $60 \mathrm{~dB}$ SPL essentially replicated those of Warm and Foulke (1970). (The exception may have arisen because, in the present experiment, noise was used as a stimulus and hence transients were not produced in response to sudden switching.)

The difference between the effects of lower and higher noise levels can be fairly easily explained as follows. Note first that reaction times to the end of the lower and higher level noise showed much the same pattern of dependency on rise-decay time: the difference, then, is essentially in the reaction times to the start of the noise. A consequence of defining the start of the noise from the time at which $10 \%$ of maximum voltage was attained is that the subject could obtain auditory information before the defined start of the noise: for the $40-\mathrm{dB}$ SPL noise, the $10 \%$ point corresponds to a level of $20 \mathrm{~dB}$ SPL, but for the 60-dB SPL noise, the $10 \%$ point corresponds to a level of $40 \mathrm{~dB}$ SPL. It would appear more sensible to define the start of the noise from a point of fixed near-threshold sound pressure level rather than from a point defined as a percentage of the maximum RMS voltage level. For this fixed point, $20 \mathrm{~dB}$ SPL seems a good choice, because (1) it already forms a fixed point for the 40-dB SPL noise condition and (2) it is a reasonable approximation to the threshold level for noise in normal-hearing subjects (Irwin, Hinchcliff, \& Kemp, 1981; Kemp, 1979; Miller, 1948). The 20-dB SPL point is reached, for the $60-\mathrm{dB}$ SPL noise, when the envelope voltage is $1 \%$ of the maximum attained.

A sensible definition of the end of the noise, however, involves different considerations. Because Weber's law holds well for noise (Maiwald, 1967; Raab, Osman, \& Rich, 1963), it seems appropriate to define the end of the noise either as the point in time at which a certain percentage of its average intensity has been reached or as the point at which a given decibel drop has occurred. The definition already chosen (see Method) fulfills this requirement; moreover, a drop to $90 \%$ of maximum voltage corresponds to a fall of $0.9 \mathrm{~dB}$, a value very close to the $1 \mathrm{~dB}$ found by Maiwald to be the approximate value of the difference threshold for noise.

Consideration of the definitions of the start and end

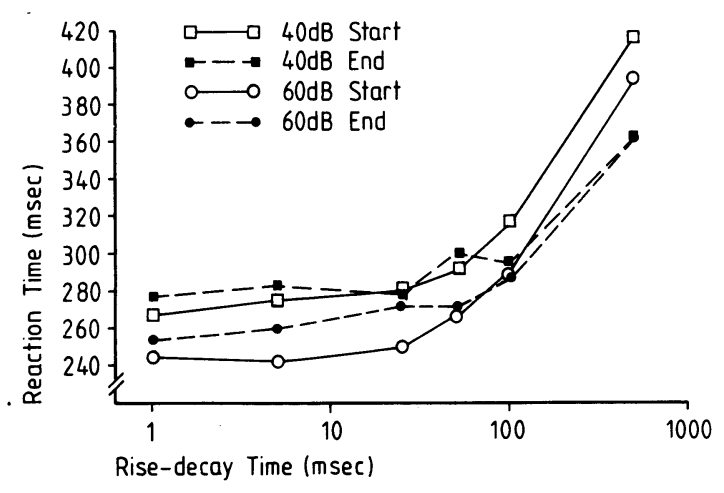

Figure 2. of the noise has led to a redefinition of the start of the 60-dB SPL noise, but has left the other event definitions unchanged. Figure 2 portrays the results obtained under this change of definition. Analysis of variance yielded significant effects of level $[F(1,9)=94.7, p<.01]$ and rise-decay time $[F(5,45)=218.7, p<.01]$. The substantial and expected dependency of reaction time on level and rise-decay time appears again in Figure 2. There were two statistically significant interactions: level $\mathrm{x}$ event type $[F(1,9)=20.4, p<.01]$ and event type $x$ risedecay time $[F(5,45)=7.1, p<.01]$. The former indicates that the effect of level in decreasing reaction time is more pronounced for start than for end events. This result is not unexpected: the effects of increased level are not mediated by Weber's law for the start of noise in the way that they are for its end.

The major consequence of the redefinition of the start of the noise is that, for both noise levels, the reactime to the start of the noise was faster than that to its end at short rise-decay times but slower at long risedecay times. A further consequence is the presumption that Warm and Foulke (1970) defined the start of the tone from a point in time occurring after audible sound was first produced. Their report, however, sheds no light on this question.

\section{CONCLUSIONS}

The following conclusions may be drawn: (1) Particularly in reaction time experiments employing relatively long rise-decay times, the definition of the start and end of the acoustic stimulus needs careful consideration. (2) Reaction time to both the start and end of noise decreased with increasing noise level and increased with increasing rise-decay time. Thus, previously reported results are generalized from tonal stimuli to noise and from responses to the start of sound to responses to its end. (3) Reaction time to the start of noise was faster than that to its end if short rise-decay times were employed, but the reverse was true if long rise-decay times were used.

\section{REFERENCES}

Emmerich, D. S., Pitchford, L. J., \& Becker, C. A. Reaction time to tones in tonal backgrounds and a comparison of reaction time to signal onset and offset. Perception \& Psychophysics, 1976. 20, 210-214.

Goldstone, S. Reaction to onset and termination of lights and sounds. Perceptual and Motor Skills, 1968, 27, 1023-1029.

Grier, J. B. Auditory reaction time as a function of stimulus intensity and rise time. Psychonomic Science, 1966, 6, 307-308. (a)

Grier, J. B. Reaction time to "tone off." Psychonomic Science, 1966, 5, 385-386. (b)

IRwIN, R. J., HinCHCLIFF, L. K., \& KEMP, S. Temporal acuity in normal and hearing-impaired listeners. Audiology, 1981, 20, 234-243.

KЕмг, S. Auditory-nerve and energy detection models of temporal summation in hearing: $A$ theoretical and experimental investigation. Unpublished PhD thesis, Auckland University, 1979.

KEMP, S., \& IRwin, R. J. Reaction time to the start and end of weak signals in noise. Psychological Research, 1979, 40, 367-376.

Maiwald, D. Die Berechnung von Modulationsschwellen mit Hilfe eines Funktionsschemas. Acustica, 1967, 18, 193-206.

Miller, G. A. The perception of short bursts of noise. Journal of the Acoustical Society of America, 1948, 20, 160-170.

Murray, H. G. Stimulus intensity and reaction time: Evaluation 
of a decision theory model. Journal of Experimental Psychology, 1970, 84, 383-391.

RAAB, D. H., Osman, E., \& Rich, E. Effects of waveform correlation and signal duration on detection of noise bursts in continuous noise. Journal of the Acoustical Society of America, $1963,35,1942-1946$.

Santee, J. L., \& Kohfeld, D. L. Auditory reaction time as a function of stimulus intensity, frequency and rise time. Bulletin of the Psychonomic Society, 1977, 10, 393-396.
Sticht, T. C., \& Foulke, E. Reaction time to the onset and offset of electrocutaneous stimuli as a function of rise and decay time. Perception \& Psychophysics, 1966, 1, 361-365.

WARM, J. S., \& Foulke, E. Effects of rate of signal rise and decay on reaction time to the onset and offset of acoustic stimuli. Perception \& Psychophysics, 1970, 7, 159-160.

(Manuscript received for publication March 14, 1983.) 